\title{
Effect of biomass pretreatment on the product distribution and composition resulting from the hydrothermal liquefaction of short rotation coppice willow
}

\author{
I.F. Grigoras, R.E. Stroe, I.M. Sintamarean, L.A. RosendahI*
}

Abstract: A major challenge for the implementation of hydrothermal liquefaction (HTL) as a continuous process is the formulation of lignocellulosic feedstock, which is prone to phase separation into water and biomass parts when pressurized. One approach to remedy such phase separation is to reduce the dry matter content; however, as this approach is detrimental to process cost efficiency, designing an appropriate pretreatment step to ensure pumpability at high dry matter content is preferable. This paper evaluated the effect of various pretreatment methods on product distribution and composition resulting from the HTL of willow and proposes short rotation coppice as an alternative biomass feedstock for biofuels production. Alkaline-thermal pretreatment, besides making high dry matter pumpable feedstock slurries, also led to an increase in the production of the bio-crude product with an oxygen content lower than 8 wt.\% and a higher concentration of aromatics and phenolic compounds. 


\section{Introduction}

Hydrothermal liquefaction (HTL) is a thermochemical process that utilizes the properties of water at temperatures higher than $553 \mathrm{~K}$ and pressures of up to $35 \mathrm{MPa}$ (Elliott et al., 2015; Toor et al., 2011) to convert the main components of lignocellulosic materials (cellulose, hemicellulose and lignin) into its monomers through a series of complex chemical reactions, which are still not fully understood. This process is still under development and will have to compete with a crude oil price below US \$50 per barrel and unconventional oil and gas as well as other biofuel technologies. The environmental effects of second-generation (2G) biofuel technologies are internalized in the cost, whereas those of fossil fuels are not; thus, competition arises with other biofuel technologies. In this context, a continuous HTL process is a prerequisite for commercializing the technology.

For this purpose, the efficient preparation of biomass feedstocks with high dry matter content has been a challenge because of the difficulty of injecting the biomass feedstock into the HTL reactors at high pressures (Daraban et al., 2015; Elliott et al., 2015; Lappa et al., 2016). The phase separation between the biomass particles and aqueous phase can be avoided by reducing the particle size to microns with or without the addition of an oil component (Berglin et al., 2012; Daraban et al., 2015); however, such fine milling of woody biomass is energy intensive. To avoid excessive reductions in the particle size, chemical pretreatment can be 
employed to destroy the lignocellulosic matrix and dissolve the substrate. Acid, neutral or alkaline pretreatment can be applied for this purpose.

Liquid hot water (LHW) pretreatment or auto-hydrolysis is usually carried out in aqueous medium at $453-473 \mathrm{~K}$ aiming to break down the hemicellulose into sugars and organic acids to create a paste product with 20-25\% biomass loading (Goudriaan and Naber, 2015; Goudriaan and Peferoen, 1990). The produced organic acids decrease the $\mathrm{pH}$ of the liquid phase, thus favoring the degradation of the hemicellulose and sugar conversion into furfurals. Lignin rearrangements in the lignocellulosic matrix and reductions in cellulose degree of polymerization and crystallinity after this kind of pretreatment have also been reported (Mosier, 2013; Mosier et al., 2005). However, these changes are not sufficient to dissolve the woody biomass to an extent that allows for the formation of homogeneous pumpable feedstock slurries, which is why Shell failed to produce a pumpable woody feedstock for the hydrothermal upgrading (HTU) process.

Using acids as a medium to create pumpable feedstocks is not desired from a material cost perspective (high amounts of alkalis are required to neutralize the acidic feed) and due to the mismatch of reaction conditions between the pretreatment and HTL conversion stage (e.g., the LBL process developed by the Lawrence Berkeley National Laboratory). In contrast, alkaline conditions are imperative to enhance bio-crude yield and reduce char formation by avoiding the re-polymerization of the cellulose and hemicellulose degradation products. Alkaline hydrothermal 
(AHT) pretreatment has a considerable impact on biomass, dissolving the lignocellulosic substrate and converting the biomass-water mixture into a pumpable paste or liquid (Daraban et al., 2015). Under alkaline conditions, all lignocellulosic biomass components are affected. The main decomposition products are organic acids resulting from carbohydrates and phenolic compounds derived from lignin (Hendriks and Zeeman, 2009; Ramirez et al., 2013; Sjostrom, 1993).

In this study, LHW and AHT pretreatments have been proposed as methods to prepare pumpable wood pastes to address the pumpability issue in HTL continuous systems. Thus, the effect of biomass degradation on the ensuing HTL product distribution and chemical composition is investigated. To this end, willow has been subjected to pretreatment methods—-neutral and alkaline media with and without thermal treatment-and then tested under supercritical water conditions. The main objective in this work is to identify an effective pretreatment method aligned with the overall process scheme for continuous HTL processing, favoring the production of bio-crude characterized by chemical compounds that can be easily upgraded in a subsequent refinery stage.

Short rotation coppice (lignocellulosic biomass), including willow, miscanthus, poplar or switchgrass could be considered alternative feedstocks to wood for biofuels production. These perennial crops do not require a considerable amount of fertilizer to grow; thus, the land used is protected. The crops could also be used for wastewater cleaning, balancing of nutrients or phyto-extraction of hazard metals, such as 
cadmium (Cd) from agricultural soils (Greger and Landberg, 2015). Additionally, willow can be harvested on cycles as short as two to three years and represents a suitable crop to be grown on marginal lands. A fast growing, low fertilizer intake and high density biomass such as willow would represent an important biomass feedstock for biofuels production and it is the aim of this study to investigate how this short rotation crop could be formulated (pre-treated) for high-pressure pumpability purposes and delivery of a petroleum substitute product through HTL.

\section{Materials and Methods}

\subsection{Materials}

Samples of willow-genus Salix —were collected at NyVraa Bioenergi I/S in Tylstrup, Denmark. Trees were harvested at two years old and were cut into chips with a width of $14 \mathrm{~mm}$ and length of $20-70 \mathrm{~mm}$. The elemental and ash content of willow is given in Table 1. All chemicals used in this study, including sodium hydroxide $(\mathrm{NaOH})$, acetone, dichloromethane (DCM) and the gas standards were purchased from Sigma Aldrich. Distilled water was used in all experiments.

\subsection{Pretreatment}

Four pretreatment methods were investigated in this work, which resulted in four pretreated feedstocks for the HTL processing. Feedstock A represented a mixture of milled willow and water, whereas Feedstock $C$ was a mixture of milled willow and $\mathrm{NaOH}$ solution with a mass fraction of 
0.1. Willow was milled to a particle size smaller than $0.10 \mathrm{~mm}$ using a laboratory scale FOSS Cyclotec ${ }^{\text {TM }}$ mill. Feedstocks B and D, the LHW and AHT pretreated feeds respectively, were prepared in a $400 \mathrm{~mL}$ stainless steel autoclave. In each batch, $10 \mathrm{~g}$ of willow chips were mixed with $40 \mathrm{~g}$ of water for the LHW pretreatment or with sodium hydroxide solution in the case of AHT pretreatment. The pretreatment temperature of $453 \mathrm{~K}$ was reached in $25 \mathrm{~min}$, whereupon the willow was pretreated for $2 \mathrm{~h}$. All four mixtures contained a wood mass fraction of $20 \%$ on a dry basis. The $\mathrm{pH}$ of the pretreated feedstocks was measured prior to the liquefaction process. Feedstocks $A$ and $B$ were characterized by an acidic $\mathrm{pH}(\mathrm{pH}=$ 4.00 and 3.00 , respectively), whereas Feedstocks $C$ and D were alkaline $(\mathrm{pH}=13.50$ and 12.50, respectively).

\subsection{Hydrothermal liquefaction}

HTL experiments were conducted in $10 \mathrm{~mL}$ stainless steel tubular batch reactors. In a typical run, approximately $7 \mathrm{~g}$ of prepared feedstock was loaded into the tubular reactor. The reactor was purged with nitrogen $\left(\mathrm{N}_{2}\right)$ for air removal, pre-pressurized with approximately $2 \mathrm{MPa} \mathrm{N}_{2}$ and then inserted into a pre-heated fluidized sand bath. The initial pressure was necessary to maintain all compounds in the liquid phase while heating the reactors from room temperature to the desired HTL conditions; however, it does not affect the final pressure in the reactor. After each experiment, the reactor was removed from the fluidized sand bath and quenched in ice water. All experiments were 
performed in triplicate. Each feedstock was converted at $673 \pm 2 \mathrm{~K}$ and $32-33$ MPa under three HTL reaction times: 0, 10 and 20 min.

After the HTL conversion, the gas products were collected in small bottles fitted with crimp butyl caps similar to the ones described by (Glatzel and Well, 2008). After gas collection, the water phase was poured out of the reactor and any solids were filtered out. Afterwards, the reactor was thoroughly rinsed with acetone to collect a mixture of bio-crude and solids. The mixture was further filtered for separation of the solids, which were dried at $378 \mathrm{~K}$ for $24 \mathrm{~h}$. The biocrude-acetone mixture was evaporated in a rotary evaporator to remove the acetone. Any component characterized by a boiling point below the atmospheric equivalent temperature (AET) of $330 \mathrm{~K}$ was lost from the bio-crude during the solvent evaporation. The remaining product-a mixture of bio-crude and residual water-was further mixed with hydrochloric acid $(\mathrm{HCl})-5.12$ vol.\%— and centrifuged to separate the oil phase from the residual water (and also to break down any formed emulsions). Following centrifugation, the residual water and acid were separated from the bio-crude gravimetrically. Thus, the HTL process produced four products: the bio-crude, aqueous phase, solids and gases.

The yields of the bio-crude and solid fractions were calculated with equations (1) and (2), respectively. The gas yield was quantified by GC analysis. Equations (3) and (4) represent the carbon and hydrogen balance, respectively. In these equations, $C_{\text {product }}$ and $H_{\text {product }}$ represent the amount of carbon and hydrogen (in grams), respectively, determined by using the elemental content measured according with the applied analyses as 
described in the next section. The carbon and hydrogen balances reveal how much of the initial carbon and hydrogen, respectively, present in willow end up in the HTL products. The $C w t . \%$ and $H w t . \%$ for the aqueous phase was not calculated.

Yield $_{\text {biocrude }}=$ Weight $_{\text {biocrude }} /$ Weight $t_{\text {dry willow }} \times 100$

Yield $_{\text {solids }}=$ Weight $_{\text {solids }} /$ Weight $t_{\text {dry willow }} \times 100$

$C w t . \%=\left(C_{\text {product }} / C_{\text {feed }}\right) \times 100$

$H w t . \%=\left(H_{\text {product }} / H_{\text {feed }}\right) \times 100$

\subsection{Feed and Product Analyses}

The elemental content of willow was measured according to the ASTM D5373 method, whereas the ash content was determined following the ASTM D3174 method.

Components in the gaseous product such as carbon dioxide $\left(\mathrm{CO}_{2}\right)$, methane $\left(\mathrm{CH}_{4}\right)$, propane and butane, were analyzed on an Agilent model 7890A gas chromatograph (GC) equipped with a thermo-conductivity detector (TCD) and two columns-HP-Molesieve (30m long, 0.32mm ID,

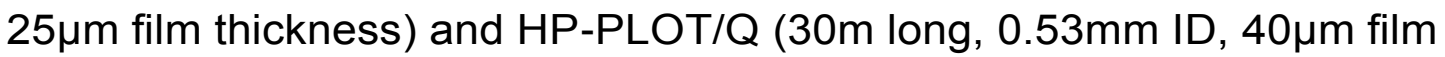
thickness). Hydrogen $\left(\mathrm{H}_{2}\right)$ was separated in a ShinCarbon ST column installed on a Chrompack CP9002 GC-TCD. The detector and injector temperature was $573 \mathrm{~K}$, whereas the oven temperature was constant at $353 \mathrm{~K}$. The mole fractions of the gas products were determined from single point calibration obtained by analysis of gas standards of known composition. 
The aqueous phase and bio-crude samples were extracted with dichloromethane (DCM) and analyzed by gas chromatography-mass spectrometry (GC-MS). No internal standard was used to calibrate these samples. The peak area (\%) was used instead to represent the comparative and semi-quantitative content of each component in the biocrude and aqueous phase samples. The applied GC-MS was a Thermo Scientific Trace 1300 GC equipped with a single quadrupole MS. The analyses were performed in electron ionization (EI) mode, with compounds being separated in an Agilent HP 5-ms column (30 m long, $0.25 \mathrm{~mm}$ ID, $0.25 \mu \mathrm{m}$ film thickness). The detector temperature was 573 $\mathrm{K}$, while the injector was held at $553 \mathrm{~K}$ and a split ratio of 20:1 was applied. The following program was used for analyzing the HTL products: $313 \mathrm{~K}$ isothermal for $3 \mathrm{~min}, 313$ to $598 \mathrm{~K}$ at a temperature ramp of 5 $\mathrm{K} / \mathrm{min}$, and held at $598 \mathrm{~K}$ for $3 \mathrm{~min}$. The liquid phase resulting from the thermal pretreatments (LHW and AHT) of willow was analyzed by GC-MS using the same program and solvent for extraction as in the case of the HTL aqueous phase. The detected compounds were identified by searching for compounds with similar mass spectra in the 2014 National Institute of Standards and Technology (NIST) library.

Moreover, infrared (IR) data was obtained at room temperature for the solid products on a Perkin-Elmer Spectrum One instrument coupled with a horizontal ATR accessory (MIRacle, Pike Technologies) with a diamond crystal as the internal reflection element. 
Furthermore, the elemental analysis of bio-crude oils was performed according to the ASTM D5291 procedure, whereas the elemental content of solids was determined following the ASTM D5373 method. The percentages of residual water were measured in the bio-crude samples by Karl Fischer titration following the ASTM E203 method.

Additionally, the ash composition of solid samples was analyzed by inductively coupled plasma-optical emission spectroscopy (ICP-OES). The small sample size was the reason for not being able to carry this type of analysis for bio-crude samples.

\section{Results and discussion}

Pretreatment efficiency on biomass deconstruction depends on the slurry $\mathrm{pH}$ and pretreatment temperature. The changes occurring in the raw biomass structure while subjected to the four pretreatment methods considered in this study are addressed in Section 3.1. A first novelty in this study is to bring to light what kind of chemical compounds should be expected in the HTL products after a certain pretreatment was applied to the biomass feedstock prior to the HTL process and how easily is it to upgrade the resulting bio-crude to a finished fuel. More details in Sections 3.2 and 3.3. A reaction time study was conducted to determine how the product distribution and composition is affected, as this influence becomes critical for process optimization when processing the biomass in continuous mode (Section 3.4). 


\subsection{Effect of $\mathrm{pH}$ and thermal treatment on the raw biomass prior to HTL}

The feedstock slurries prepared for HTL conversion varied in composition according to the pretreatment method employed. There were no changes in the biomass structure or composition occurring in Feedstock $A$ with the exception of some possible dissolution of extractives, which could explain the decrease in $\mathrm{pH}$. The $\mathrm{pH}$ of this slurry decreased from neutral to a value of approximately 4.00 only by soaking the willow particles in distilled water. This value might vary as the raw biomass changes according to a study published in Nature (Campbell and Bryant, 1941), becoming either more acidic or closer to neutral $\mathrm{pH}$ depending on the type of species involved.

When heat was applied to such a feedstock (LHW pretreatment)Feedstock B-sugars and derived organic acids were generated by hemicellulose hydrolysis. Qualitative GC-MS data revealed the production of furfurals and 5-methyl-2-furaldehyde, which have likely resulted from the hydrolysis of hemicellulose and subsequent reactions involving its monomers, including xylose, mannose, and glucose. Phenol, 2-methoxyphenol and 2,6-dimethoxy-phenol have been identified as well, which may be a result of lignin degradation during the pretreatment. Complete dissolution of lignin was not achieved in Feedstock $\mathrm{B}(\mathrm{pH}=3.0)$, likely because of the low pretreatment temperature or reaction time or even the acidic conditions, which were not strong enough to break down the lignin backbone. 
In contrast, the dissolution of lignin and polysaccharides was promoted in alkaline conditions. In the case of cold alkaline pretreatmentFeedstock C-some of the hemicellulose compounds dissolved, resulting in a feed with increased viscosity and water-holding capacity.

The most dramatic alteration of the willow structure occurred during the AHT pretreatment, in which approximately $70 \%$ of the initial solid matter was dissolved in the alkaline solution. The result was a slurry with a paste consistency and a complex mixture of biomass-derived compounds. Hemicellulose and cellulose generated a range of organic acids, whereas lignin provided a large spectrum of phenolic compounds, including 2methoxy-phenol, 4-ethyl-2-methoxy-phenol, 2,6-dimethoxy-phenol, and 5tert-1,2,3-benzenetriol.

The major components of biomass started to degrade under alkaline and thermal pretreatment at $453 \mathrm{~K}$ with the formation of monomers and smaller compounds depending on the $\mathrm{pH}$ of the medium (either acidic or basic). The resulting compounds are expected to undergo various conversion pathways depending largely on the reaction medium. These variations make it important to study the effect of a pretreatment method when processing biomass for biofuel production; this effect on product distribution and composition is addressed in the following two sections. 


\subsection{Effect of slurry $\mathrm{pH}$ and thermal treatment on the HTL product distribution and elemental content}

HTL conducted in an acidic medium promotes char formation and suppresses bio-crude production. These effects were also observed in this study as the bio-crude yield resulting from the conversion of Feedstock $\mathrm{B}(\mathrm{pH}=3.00)$ was the lowest (see Table 3$)$. This low conversion could be explained by the increased concentration of organic acids produced during the thermal pretreatment, which favored formation of gases and solids (see Table 3). Unlike Feedstock B, Feedstock A generated a higher bio-crude yield, likely because of smaller particle size of biomass, which increased the surface area for water attack and also due to a decreased concentration of organic acids.

In contrast, alkalis, such as $\mathrm{NaOH}$, or potassium carbonate $\left(\mathrm{K}_{2} \mathrm{CO}_{3}\right)$, are used in the HTL process as char reduction agents and bio-crude yield enhancers (Karagoz et al., 2005; Yin et al., 2011), promoting retro-aldol reactions/cleavage and condensation reactions (Mazaheri et al., 2010) and preventing re-polymerization reactions (Li et al., 2015; Liu et al., 2014; Minowa et al., 1998). In Table 3, the solid yield for the results from alkaline pretreatment was higher if compared to the results from the acidic study as it contained the ash which was formed mainly of $\mathrm{Na}$. Analysis of ash by ICP included in Table 1 confirmed this fact. More than $23 \mathrm{mg} / \mathrm{g} \mathrm{Na}$ was measured in the ash of solids resulting from Feeds $C$ and $D$ as compared to only $0.15-0.18 \mathrm{mg} / \mathrm{g}$ in the case of solids from Feeds A and B, most likely derived from willow (1.9 wt.\% ash). Some of the Na derived 
from the alkaline agent is expected to end up in the bio-crude composition as well, but no ICP analysis can support this statement, as the sample size for these samples was not enough to conduct this type of analysis. The AHT pretreatment also led to a decrease in the gas yield from approximately $19.6-24.5$ wt.\% to $14.6-17.8$ wt.\% (Table 3).

Feedstock D produced a bio-crude yield higher than 40 wt.\%, which could be explained by the increased reaction rates during the HTL process due to biomass dissolution. Additionally, the liquid homogeneous phase created by this pretreatment method could also have played a role in the enhancement of the oil productivity and be related to the decomposition of lignin to low-molecular-weight (LMW) compounds in the liquid phase. This alkaline solution might have shifted the reactions toward the formation of oil compounds rather than going under condensation or re-polymerization reactions. However, this was not the case of Feedstock $\mathrm{C}$, as the bio-crude yield was about the same as it was in the case of Feedstock B. This might be related to the initial slurry alkalinity as reported by (Yin et al., 2011), which can change the reaction pathway during hydrothermal conversion such that the reactions can involve both alkaline and acidic pathways. Thus, an increase in the alkalinity of solutions might lead to a decrease in the bio-crude yield. Moreover, the solvent used in the extraction of the liquid product is important because the polarity of certain components, such as hydroxymethyl-furfural (HMF), is weaker than those of e.g. carboxylic acids, and thus, certain components are more soluble than others and can be more 
easily extracted as bio-crude. The applied extraction solvent-in this case, acetone-could have had an influence on the bio-crude yields. The aqueous phase comprised of many compounds similar to the ones in the bio-crude product (see section 3.3). Furthermore, some low molecular components with boiling points lower than $330 \mathrm{~K}$ might had been lost from the bio-crude during solvent evaporation. The applied separation procedure might have had an effect on bio-crude yields as well.

An alkaline reaction medium (attached to a thermal treatment or not of the raw biomass) is preferable when comparing the elemental content in the bio-crudes and solids (see Table 1). The carbon content in the biocrudes increased from 69 wt.\% (Feed A and B) to 75 wt.\% (Feed C and D), whereas in the solids decreased from 70 wt.\% (Feed A and B) to 3025 wt.\% (Feed C and D). Solid formation occurs during the HTL process because of re-polymerization and condensation reactions. Carbohydrate conversion into solids is enhanced in acidic media and suppressed in the presence of $\mathrm{NaOH}$. The hydrogen content in these products followed a similar path. The nitrogen content does not depend on the reaction medium and/or pretreatment method. Nitrogen content in bio-crude is dependent on the biomass composition, as it is derived from willow. Carbon and hydrogen balances showed that about $60 \%$ and $65 \%$ of the initial carbon and hydrogen, respectively present in willow was transferred to the bio-crude when subjected to an alkaline and thermal treatment. The situation was similar in the case of bio-crude derived from Feed A. 
Performing an $\mathrm{AHT}$ pretreatment on the raw biomass to produce a pumpable feedstock slurry for continuous HTL seems to be a plausible option because it enhances the bio-crude productivity. However, recycling of sodium from the solid fraction has to be considered for reduction of processing costs. Willow pretreatment by fine milling led to similar results, so a study on processing costs could reveal which one of these two options is better for continuous HTL. In addition, the effect of such a pretreatment on the composition of bio-crude is of high interest in the upgradability of the product to a finished fuel; this topic is discussed in the next section.

\subsection{Effect of slurry $\mathrm{pH}$ and thermal treatment on the HTL product composition}

$\mathrm{GC} / \mathrm{MS}$ results of the bio-crude fractions revealed a significant difference in chemical composition as a function of the reaction medium. Thus, the volatile compounds detected by GC-MS were distributed in the same classes of compounds, but their relative abundance varied with the reaction medium, and in the case of experiments conducted in acidic medium, their relative abundance varied significantly with the thermal treatment of the raw biomass as well.

The majority of the chemical compounds identified in the bio-crude products were oxygenates: ketones, phenols and alcohols. More details about the nature of these compounds is given in Table 2 . 
A major difference in composition was represented by the near complete disappearance of the esters from the composition of bio-crude products resulting in an alkaline medium. This might be related to the neutralization of the organic acids resulting from hydrolysis of polysaccharides during alkaline pretreatment. The di-ester of terephthalic acid was identified in the composition of bio-crude products obtained from conversion of Feeds A and B. The presence of these high molecular weight (HMW) organic esters is expected to increase the total acid number (TAN) of the bio-crude products. Such compounds are undesirable because of the difficulty of storing products with a high TAN number and because of the downstream processing; de-esterification of two ester groups is typically achieved in two steps (Furimsky, 2013). Another effect of the reaction medium was an increase in the relative abundance of phenolic compounds and alcohols in bio-crude products as the $\mathrm{pH}$ of the feed slurry became alkaline. This fact was also noticed by (Ogi et al., 1985) and (Akhtar et al., 2010). Such a behavior would be preferable as the formation of heavy organic acids is inhibited under these conditions. Furthermore, alcohols and ketones are easy to upgrade because they require only one hydrogen molecule to convert into hydrocarbons. Most of the phenolics in bio-crude samples were substituted phenols, and they are more difficult to handle during a hydrodeoxygenation process compared to simple phenols (Furimsky, 2013).

The $\mathrm{pH}$ measurements of the $\mathrm{HTL}$ aqueous products indicated that a large fraction of the alkaline agent $(\mathrm{NaOH})$ added to the feedstock prior to 
conversion was found in the water phase product; the $\mathrm{pH}$ values for cases C and D were 9.65 and 9.50, respectively, whereas the values for cases A and $B$ were 3.75 and 3.95 , respectively. This result indicates that any organic acid produced after the pretreatment process was neutralized by the $\mathrm{NaOH}$. This fact was also confirmed by the GC-MS analysis, where the distribution of the classes of compounds depended on the reaction medium and the thermal treatment applied to the raw biomass. The distribution showed a slightly different classification of the compounds as compared to the bio-crude product, comprising of alcohols, ketones and aldehydes, phenols, furans and carboxylic acids (see supplemental material).

The aqueous products resulting from conversion under an acidic medium (Aqueous phase Feed A) were rich in furans, acids, ketones and phenolic compounds, in contrast to the products resulting from alkaline conversion (Aqueous phase Feed C), which were predominantly composed of ketones and small percentages of alcohols and phenolic compounds. This result highlights that under the action of an alkaline agent, furfurals and acids, which are typically formed in acidic medium, might be directed to form ketones and alcohols. The phenolic compounds were also affected by alkalinity; their percentage area decreased in aqueous products obtained from alkaline conversion. Moreover, the aldehydes present in Aqueous phase Feed A vanished when an alkaline agent was employed in the HTL process. Their disappearance could be explained by the Cannizzaro reaction, by which they form alcohols 
through disproportionation. When a thermal treatment was applied to the feedstock slurry (Feedstock B) prior to HTL conversion, the furan abundance in the aqueous product increased while the relative abundance of phenolic compounds and ketones decreased, suggesting an enhancement in the hydrolysis of hemicellulose under the thermal treatment to form furfurals.

A challenge in the continuous processing of such biomass feedstock slurries to produce biofuels would be to optimize the process such that the majority of these organic compounds in the HTL aqueous products become part of the bio-crude product. Gravimetrical separation of the aqueous and bio-crude products or by using membranes would be the preferable options from an industrial point of view and not by employing a solvent, which would increase the operation costs.

Another important aspect in terms of continuous operation is the recycling of the water phase because it contains the majority of the alkaline agent. Zhu et al. (Zhu et al., 2015) demonstrated that aqueous phase recirculation enhanced the bio-crude yield when converting barley straw through HTL in a batch process but also increased the solid residues with the number of recycling cycles, highlighting that the dissolved organic compounds in the aqueous phase could play a role in re-polymerization reactions to form solids.

Pedersen et al.(Pedersen et al., 2016) demonstrated that recirculation of the aqueous phase can also impact the quality of the bio-crude in terms of the effective carbon-to-hydrogen $(\mathrm{C} / \mathrm{H})$ ratio, which increased when 
processing a mixture of aspen wood and glycerol continuously under supercritical water conditions. Water is a commodity that is expected to become expensive in the future; thus, recirculation of aqueous products in such a process and recovery of the alkaline agent are necessary to minimize operation costs.

The gaseous products obtained from conversion of all four feedstocks consisted mainly of $\mathrm{CO}_{2}$ (see Figure 1). $\mathrm{CO}_{2}$ accounted for more than 75 $\%$ of the produced gases in the case of gas derived from conversion of feedstocks in acidic medium. The higher concentrations of $\mathrm{CO}_{2}$ in the gas produced from Feed $A$ and Feed $B$ indicate that the acidic medium promoted decarboxylation reactions involving organic acids derived from the lignocellulosic biomass. (Eager et al., 1981) found that $\mathrm{CO}_{2}$ could be released from carbohydrates during the formation of certain intermediates. These reactions appear to be inhibited when a thermal treatment is applied (Feedstock B); the solid yield increased significantly, indicating that re-polymerization reactions are favored. Residual carbon could also be formed through the disproportionation of carbon monoxide (CO) into carbon and carbon dioxide $\left(2 \mathrm{CO} \leftrightarrow \mathrm{C}+\mathrm{CO}_{2}\right)$.

Analysis of the gas phase produced in alkaline conditions illustrates that dehydrogenation reactions are enhanced. The presence of higher concentrations of hydrogen in the gas phase obtained from alkalinepretreated feeds might be due to the higher fractions of $\mathrm{CO}$ generated and converted to $\mathrm{H}_{2}$ and $\mathrm{CO}_{2}$ through the water gas shift reaction. Ethane, 
ethylene, propylene and $\mathrm{CO}$ are other gases expected to result from the HTL process, but they could not be quantified in this study.

The IR data of solid fractions showed that the peaks for the $\mathrm{C}-\mathrm{C}$ ring stretch $\left(1570 \mathrm{~cm}^{-1}\right), \mathrm{C}-\mathrm{O}$ stretch $\left(1420 \mathrm{~cm}^{-1}\right)$ and $\mathrm{C}-\mathrm{C}$ ring bend or aromatic $\mathrm{C}-\mathrm{H}$ bend $\left(870 \mathrm{~cm}^{-1}\right)$ were more intense in the case of solid particles procured from alkaline feedstock slurries. All of these bands indicate the aromatic character of the 'alkaline' solids.

\subsection{Effect of reaction time on the HTL product distribution and composition}

Reaction time plays an important role in the optimization of the HTL process. The results indicated that a longer reaction time indeed affects the product distribution: the bio-crude production was negatively affected, whereas the solid and gas production increased. This result demonstrates that re-polymerization and gasification reactions are favored at longer reaction times. As the reaction time is prolonged, water-soluble compounds are also subjected to re-polymerization and/or gasification reactions. The observation that the bio-crude production is highest at shorter reaction times (in this case, $0 \mathrm{~min}$ ) demonstrates that the compounds from the biomass convert into oil compounds first and then either become soluble in the aqueous phase or produce the gases and solids with the increasing reaction time. Thus, it will be important from an optimization perspective to identify the stage at which these compounds 
start to become soluble into the aqueous phase or react to form gases and solids.

The change in composition with increasing reaction time will impact the subsequent step (upgrading); thus, knowing the types of compounds in the bio-crude becomes significant. The elemental composition of the biocrude products exhibited a slight increase in the hydrogen contents at longer reaction times, whereas the oxygen content was reduced. As addressed in the previous sections, hydro-deoxygenation is a preferred route in the upgrading stage of the bio-crude products. As a result, the occurrence of such reactions during HTL conversion is desired.

In the case of solids, the analysis of elemental distribution did not suggest that the reaction time had any impact; the composition remained fairly constant. The IR spectra exhibited peaks for the same functional groups as explained in the previous section.

The final $\mathrm{pH}$ of aqueous products was not influenced by the reaction time either. The composition of gaseous products was influenced by the reaction time, as the contents of all gas components increased with longer reaction times in all cases (see Figure 1). Carbon and hydrogen balance showed that as the reaction time was prolonged, more of the initial carbon and hydrogen in the biomass was transferred to solids and gases.

Compared to all other gas components identified by GC analysis, propane was not formed at Omin, or the concentration was so low that the equipment could not detected. Propane might have likely formed at longer reaction times only. 
In terms of composition, a longer reaction time had an influence on the component distribution. If the relative abundance for alcohols and alkanes increased in the case of bio-crude samples resulting in an acidic medium, an opposite effect was observed for the samples derived in an alkaline medium. A similar effect was observed for phenolic compounds and ketones. In the case of organic compounds in aqueous phase derived from Feedstocks $\mathrm{A}$ and $\mathrm{B}, \mathrm{a}$ longer reaction time favored the formation of ketones and acids while the relative abundance for furans, aldehydes, alcohols and phenolics decreased. In the case of water soluble organics derived from Feedstocks $C$ and $D$, the reaction time was only observed to impact the relative abundance of ketones, which decreased, whereas the relative abundance for alcohols and phenolics increased.

From a bio-refinery perspective, a shorter reaction time during the HTL process is preferable as the bio-crude production is enhanced, but it might negatively influence the quality. Having a process that can convert biomass residues in short reaction times would be ideal if the bio-refinery was placed adjacent to the petroleum refinery so that the liquid product being separated from the residual streams (gases and solids) could be directly transferred to the upgrading unit in the petroleum refinery. In such a situation, organic compounds soluble in the aqueous phase, such as furans, ketones and phenolics, could also be hydro-deoxygenated and converted into hydrocarbons, but this case will most likely be impossible to apply in the future because of many challenges that must be considered, including building the bio-refinery next to an oil refinery, 
transportation of biomass to the bio-refinery (an extremely expensive step), and efficient and immediate separation of the liquid product from the residual streams. Additionally, water is undesirable in an oil refinery.

\section{Conclusions}

The effect of biomass pretreatment on the HTL product distribution and composition was evaluated in this work to identify an effective pretreatment method of short rotation coppice aligned with the overall process scheme for continuous HTL processing. The AHT pretreatment was the most effective on the bio-crude production and composition, improving the yield and reducing the production of oxygenates, but increasing its aromatic character. Recovery of the alkaline agent from the solid and/or aqueous phases, recycling of the aqueous phase, and utilization of gaseous and solid products are challenges that remain to be addressed in the near future.

\section{Acknowledgements}

This work was financially supported by the Innovation Fund Denmark (grant \# 1305-00030B). The authors are grateful to Niels Iversen and Helle Blendstrup from the Department of Chemistry and Bioscience at Aalborg University, Section for Environmental Technology for their support with the GC analysis of the gas phases, as well as to Federica Conti from Department of Energy Technology, Aalborg University for her support with the elemental analysis. 


\section{Notes and references}

1. Akhtar, J., Kuang, S.K., Amin, N.S., 2010. Liquefaction of empty palm fruit bunch (EPFB) in alkaline hot compressed water. Renew. Energy 35, 1220-1227. doi:10.1016/j.renene.2009.10.003

2. Berglin, E.J., Enderlin, C.W., Schimidt, A.J., 2012. Review and Assessment of Commercial Vendors / Options for Feeding and Pumping Biomass Slurries for Hydrothermal Liquefaction.

3. Campbell, W.G., Bryant, S.A., 1941. Determination of pH in Wood. Nature. doi:10.1038/147357a0

4. Daraban, I.M., Rosendahl, L.A., Pedersen, T.H., Iversen, S.B., 2015. Pretreatment methods to obtain pumpable high solid loading wood-water slurries for continuous hydrothermal liquefaction systems. Biomass and Bioenergy 81, 437-443. doi:10.1016/j.biombioe.2015.07.004

5. Eager, R.L., Mathews, J.F., Pepper, J.M., Zohdi, H., 1981. Studies on the products resulting from the conversion of aspen poplar to an oil. Can. J. Chem. 59, 2191-2198. doi:10.1139/v81-316

6. Elliott, D.C., Biller, P., Ross, A.B., Schmidt, A.J., Jones, S.B., 2015. Hydrothermal liquefaction of biomass: Developments from batch to continuous process. Bioresour. Technol. 178, 147-156. doi:10.1016/j.biortech.2014.09.132

7. Furimsky, E., 2013. Hydroprocessing challenges in biofuels production. Catal. Today 217, 13-56. doi:10.1016/j.cattod.2012.11.008

8. Glatzel, S., Well, R., 2008. Evaluation of septum-capped vials for storage 
of gas samples during air transport. Environ. Monit. Assess. 136, 307311. doi:10.1007/s10661-007-9686-2

9. Goudriaan, F., Naber, J.E., 2015. Biomass to Liquid Fuels via HTU, in: Swaaij, W. van, Kersten, S., Palz, W. (Eds.), BIOMASS POWER for the World: Transformations to Effective Use. Pan Stanford Publishing Pte. Ltd., Singapore.

10.Goudriaan, F., Peferoen, D.G.R., 1990. Liquid fuels from biomass via a hydrothermal process. Chem. Eng. Sci. 45, 2729-2734. doi:10.1016/0009-2509(90)80164-A

11.Greger, M., Landberg, T., 2015. Novel Field Data on Phytoextraction: Pre-Cultivation With Salix Reduces Cadmium in Wheat Grains. Int. J. Phytoremediation 17, 917-924. doi:10.1080/15226514.2014.1003785

12. Hendriks, A.T.W.M., Zeeman, G., 2009. Pretreatments to enhance the digestibility of lignocellulosic biomass. Bioresour. Technol. 100, 10-18. doi:10.1016/j.biortech.2008.05.027

13. Karagoz, S., Bhaskar, T., Muto, A., Sakata, Y., Oshiki, T., Kishimoto, T., 2005. Low-temperature catalytic hydrothermal treatment of wood biomass: Analysis of liquid products. Chem. Eng. J. 108, 127-137. doi:10.1016/j.cej.2005.01.007

14. Lappa, E., Christensen, P.S., Klemmer, M., Becker, J., Iversen, B.B., 2016. Hydrothermal Liquefaction of Miscanthus x Giganteus: Preparation of the ideal feedstock. Biomass and Bioenergy 87, 17-25. doi:http://dx.doi.org/10.1016/j.biombioe.2016.02.008

15. Li, Z., Cao, J., Huang, K., Hong, Y., Li, C., Zhou, X., Xie, N., Lai, F., 
Shen, F., Chen, C., 2015. Alkaline pretreatment and the synergic effect of water and tetralin enhances the liquefaction efficiency of bagasse. Bioresour. Technol. 177, 159-168. doi:10.1016/j.biortech.2014.11.043 16. Liu, H.M., Wang, F.Y., Liu, Y.L., 2014. Alkaline pretreatment and hydrothermal liquefaction of cypress for high yield bio-oil production. J. Anal. Appl. Pyrolysis 108, 136-142. doi:10.1016/j.jaap.2014.05.007

17. Mazaheri, H., Lee, K.T., Bhatia, S., Mohamed, A.R., 2010. Subcritical water liquefaction of oil palm fruit press fiber in the presence of sodium hydroxide: An optimisation study using response surface methodology. Bioresour. Technol. 101, 9335-9341. doi:10.1016/j.biortech.2010.07.004

18. Minowa, T., Zhen, F., Ogi, T., 1998. Cellulose decomposition in hotcompressed water with alkali or nickel catalyst. J. Supercrit. Fluids 13, 253-259. doi:10.1016/S0896-8446(98)00059-X

19. Mosier, N., 2013. Fundamentals of Aqueous Pretreatment of Biomass, in: Wyman, C.E. (Ed.), Aqueous Pretreatment of Plant Biomass for Biological and Chemical Conversion to Fuels and Chemicals. John Wiley \& Sons, Ltd, pp. 129-143. doi:10.1002/9780470975831.ch7

20.Mosier, N., Wyman, C., Dale, B., Elander, R., Lee, Y.Y., Holtzapple, M., Ladisch, M., 2005. Features of promising technologies for pretreatment of lignocellulosic biomass. Bioresour. Technol. 96, 673-686. doi:10.1016/j.biortech.2004.06.025

21. Ogi, T., Yokoyama, P., Koguchi, K., 1985. Direct Liquefaction of Wood by Catalyst ( Part 1 ) Effects of Pressure , Temperature, Holding Time and Wood / Catalyst / Water Ratio on Oil Yield. Sekiyu Gakkaishi 28, 239_ 
245.

22. Pedersen, T.H., Grigoras, I.F., Hoffmann, J., Toor, S.S., Daraban, I.M., Jensen, C.U., Iversen, S.B., Madsen, R.B., Glasius, M., Arturi, K.R., Nielsen, R.P., Søgaard, E.G., Rosendahl, L.A., 2016. Continuous hydrothermal co-liquefaction of aspen wood and glycerol with water phase recirculation. Appl. Energy 162, 1034-1041. doi:10.1016/j.apenergy.2015.10.165

23. Ramirez, R.S., Holtzapple, M., Piamonte, N., 2013. Fundamentals of Biomass Pretreatment at Low pH, in: Wyman, C.E. (Ed.), Aqueous Pretreatment of Plant Biomass for Biological and Chemical Conversion to Fuels and Chemicals. John Wiley \& Sons, pp. 103-128. doi:10.1002/9780470975831.ch6

24. Sjostrom, E., 1993. Wood Chemistry. Fundamentals and Applications, 2nd Editio. ed. Academic Press, San Diego.

25. Toor, S.S., Rosendahl, L., Rudolf, A., 2011. Hydrothermal liquefaction of biomass: A review of subcritical water technologies. Energy 36, 23282342. doi:10.1016/j.energy.2011.03.013

26. Yin, S., Mehrotra, A.K., Tan, Z., 2011a. Alkaline hydrothermal conversion of cellulose to bio-oil: Influence of alkalinity on reaction pathway change. Bioresour. Technol. 102, 6605-6610. doi:10.1016/j.biortech.2011.03.069

27. Yin, S., Pan, Y., Tan, Z., 2011b. Hydrothermal Conversion of Cellulose to 5-Hydroxymethyl Furfural. Int. J. Green Energy 8, 234-247. doi:10.1080/15435075.2010.548888

28.Zhu, Z., Rosendahl, L., Toor, S.S., Yu, D., Chen, G., 2015. Hydrothermal 
liquefaction of barley straw to bio-crude oil : Effects of reaction temperature and aqueous phase recirculation. Appl. Energy 137, 183192. doi:10.1016/j.apenergy.2014.10.005 
Table 1 Elemental, Water and Ash Contents of Willow and HTL Products derived from conversion of willow-based feeds at a reaction time of $10 \mathrm{~min}$

\begin{tabular}{|c|c|c|c|c|c|c|c|c|c|}
\hline \multirow{2}{*}{ Properties } & \multirow{2}{*}{ Willow } & \multicolumn{2}{|c|}{ Feed A } & \multicolumn{2}{|c|}{ Feed B } & \multicolumn{2}{|c|}{ Feed C } & \multicolumn{2}{|c|}{ Feed D } \\
\hline & & Biocrude & Solids & Biocrude & Solids & Biocrude & Solids & Biocrude & Solids \\
\hline \multicolumn{10}{|l|}{ Elemental analysis (wt.\% db) } \\
\hline C & $47.9 \pm 0.03$ & $69.2 \pm 0.5$ & $69.1 \pm 2.0$ & $69.1 \pm 4$ & $76.2 \pm 4.2$ & $74.7 \pm 1.9$ & $25.7 \pm 5.6$ & $73.9 \pm 5.9$ & $30.1 \pm 3.1$ \\
\hline $\mathbf{H}$ & $6.0 \pm 0.01$ & $8.3 \pm 0.2$ & $3.8 \pm 0.6$ & 7 & $3.5 \pm 0.2$ & $9.6 \pm 0.03$ & $1.4 \pm 0.5$ & $9.2 \pm 0.1$ & $1.95 \pm 0.03$ \\
\hline $\mathbf{N}$ & $0.01 \pm 0.02$ & $1.7 \pm 0.1$ & $0.8 \pm 0.2$ & $8.8 \pm 0.1$ & $0.7 \pm 0.1$ & $1.4 \pm 0.03$ & $0.0 \pm 0.0$ & $1.4 \pm 0.2$ & $0.0 \pm 0.0$ \\
\hline & & & & $1.5 \pm 0.1$ & & & & & \\
\hline Bound water (wt.\%) & 0.00 & $11.5 \pm 5.5$ & 0.00 & $9.6 \pm 0.8$ & 0.00 & $6^{10.8 \pm 1 .}$ & 0.00 & $16.95 \pm 1.1$ & 0.00 \\
\hline Ash/Metals content (wt.\%) & $1.90 \pm 0.08$ & n.d. & 0.30 & n.d. & 0.22 & n.d. & 2.55 & n.d. & 2.69 \\
\hline Ash Composition $(\mathrm{mg} / \mathrm{g})$ & n.d. & n.d. & & n.d. & & n.d. & & n.d. & \\
\hline $\mathrm{Al}$ & & & 0.086 & & 0.034 & & 0.026 & & 0.079 \\
\hline $\mathrm{Ca}$ & & & 1.300 & & 0.639 & & 0.812 & & 1.185 \\
\hline (a) & & & 0.351 & & 0.371 & & 0.141 & & 0.807 \\
\hline r & & & 0.123 & & 0.423 & & 0.401 & & 0.522 \\
\hline $\mathrm{Ma}$ & & & 0.166 & & 0.149 & & 0.108 & & 0.166 \\
\hline y & & & 0.155 & & 0.179 & & 23.85 & & 23.347 \\
\hline $\mathbf{p}$ & & & 0.648 & & 0.251 & & 2 & & 0.667 \\
\hline & & & 0.121 & & 0.104 & & 0.171 & & 0.084 \\
\hline & & & & & & & 0.030 & & \\
\hline
\end{tabular}


Table 2 Classification of the chemical compounds detected by GC-MS in biocrude products derived from the conversion of the four feedstock slurries under supercritical water conditions (673 K, 32-33 MPa, $10 \mathrm{~min}$ ).

\begin{tabular}{|c|c|c|c|c|c|c|c|}
\hline & \multirow[b]{2}{*}{ Chemical compound } & \multirow[b]{2}{*}{ Formula } & \multirow[b]{2}{*}{ MW } & \multicolumn{4}{|c|}{ Peak area $(\%)$} \\
\hline & & & & $\begin{array}{l}\text { Feed } \\
\text { A }\end{array}$ & $\begin{array}{l}\text { Feed } \\
\text { B }\end{array}$ & $\begin{array}{l}\text { Feed } \\
\text { C }\end{array}$ & $\begin{array}{l}\text { Feed } \\
\text { D }\end{array}$ \\
\hline \multirow{4}{*}{ Alcohols } & 3-penten-2-ol & $\mathrm{C} 5 \mathrm{H} 10 \mathrm{O}$ & 86 & 2.94 & 6.85 & 4.98 & 3.39 \\
\hline & 4-methyl-2-pentanol & $\mathrm{C} 6 \mathrm{H} 14 \mathrm{O}$ & 102 & 0 & 3.09 & 2.30 & 1.75 \\
\hline & 5-octen-1-ol & $\mathrm{C} 8 \mathrm{H} 16 \mathrm{O}$ & 128 & 0.67 & 0.75 & 1.47 & 1.49 \\
\hline & 2-methyl-1-hexadecanol & $\mathrm{C} 17 \mathrm{H} 36 \mathrm{O}$ & 256 & 1.55 & 1.06 & 1.58 & 1.45 \\
\hline \multirow{16}{*}{ Ketones } & Cyclopentanone & $\mathrm{C} 5 \mathrm{H} 8 \mathrm{O}$ & 84 & 0.98 & 0.86 & 0.43 & 0.46 \\
\hline & 2-methyl-cyclopentanone & $\mathrm{C} 6 \mathrm{H} 10 \mathrm{O}$ & 98 & 0 & 1.73 & 1.77 & 1.51 \\
\hline & 4-hydroxy-4-methyl-2-pentanone & $\mathrm{C} 6 \mathrm{H} 12 \mathrm{O} 2$ & 116 & 6.47 & 1.45 & 2.78 & 2.69 \\
\hline & 3-methyl-cyclohexanone & $\mathrm{C} 7 \mathrm{H} 12 \mathrm{O}$ & 112 & 0 & 0 & 1.89 & 1.34 \\
\hline & 2-methyl-2-cyclopenten-1-one & $\mathrm{C} 6 \mathrm{H} 8 \mathrm{O}$ & 96 & 2.07 & 0.90 & 0.42 & 0.48 \\
\hline & 2,3-dimethyl-2-cyclopenten-1-one & $\mathrm{C} 7 \mathrm{H} 10 \mathrm{O}$ & 110 & 0.68 & 0.49 & 0.36 & 0.51 \\
\hline & 3-methyl-2-cyclopenten-1-one & $\mathrm{C} 6 \mathrm{H} 8 \mathrm{O}$ & 96 & 3.65 & 0 & 0 & 0 \\
\hline & 3,4-dimethyl-2-cyclopenten-1-one & $\mathrm{C} 7 \mathrm{H} 10 \mathrm{O}$ & 110 & 0.96 & 0.35 & 1.00 & 0.47 \\
\hline & 2,3-dimethyl-2-cyclopenten-1-one & $\mathrm{C} 7 \mathrm{H} 10 \mathrm{O}$ & 110 & 0.97 & 0.56 & 0.43 & 0.70 \\
\hline & 3,4-dimethyl-2-cyclopenten-1-one & $\mathrm{C} 7 \mathrm{H} 10 \mathrm{O}$ & 110 & 0.87 & 0.49 & 0 & 0.47 \\
\hline & 2,3-dimethyl-2-cyclopenten-1-one & $\mathrm{C} 7 \mathrm{H} 10 \mathrm{O}$ & 110 & 3.65 & 1.48 & 1.01 & 1.24 \\
\hline & 2,3,4-trimethyl-2-cyclopenten-1-one & $\mathrm{C} 8 \mathrm{H} 12 \mathrm{O}$ & 124 & 0.56 & 0.33 & 1.11 & 1.22 \\
\hline & 3-isopropyl-2-cyclopenten-1-one & $\mathrm{C} 8 \mathrm{H} 12 \mathrm{O}$ & 124 & 0.57 & 0.37 & 0 & 0.82 \\
\hline & $\begin{array}{l}\text { 2,3,4,5-tetramethyl-2-cyclopenten-1- } \\
\text { one }\end{array}$ & $\mathrm{C} 9 \mathrm{H} 14 \mathrm{O}$ & 138 & 0.42 & 0 & 0.55 & 0.75 \\
\hline & 3-isopropyl-2-cyclopenten-1-one & $\mathrm{C} 8 \mathrm{H} 12 \mathrm{O}$ & 124 & 0.58 & 0 & 0 & 0 \\
\hline & $\begin{array}{l}\text { 2,2-dimethylcyclohexyl methyl } \\
\text { ketone }\end{array}$ & $\mathrm{C} 10 \mathrm{H} 18 \mathrm{O}$ & 154 & 0.99 & 1.65 & 3.43 & 3.44 \\
\hline \multirow{12}{*}{ Phenols } & Phenol & $\mathrm{C} 6 \mathrm{H} 6 \mathrm{O}$ & 94 & 1.98 & 0.65 & 0.66 & 0.83 \\
\hline & m-cresol & $\mathrm{C} 7 \mathrm{H} 8 \mathrm{O}$ & 108 & 0.88 & 0.5 & 0.55 & 0.79 \\
\hline & p-cresol & $\mathrm{C} 7 \mathrm{H} 8 \mathrm{O}$ & 108 & 1.51 & 0 & 0.52 & 0.63 \\
\hline & 2-methoxy-phenol & $\mathrm{C} 7 \mathrm{H} 8 \mathrm{O} 2$ & 124 & 2.01 & 0.78 & 1.26 & 1.84 \\
\hline & 2,3-xylenol & $\mathrm{C} 8 \mathrm{H} 10 \mathrm{O}$ & 122 & 0.38 & 0.63 & 0.60 & 0.72 \\
\hline & 2-methoxy-4-methyl-phenol (creosol) & $\mathrm{C} 8 \mathrm{H} 12 \mathrm{O} 2$ & 138 & 1.25 & 1.67 & 3.04 & 2.69 \\
\hline & 2,3,6-trimethyl-phenol & $\mathrm{C} 9 \mathrm{H} 12 \mathrm{O}$ & 136 & 0 & 0 & 0.93 & 0.84 \\
\hline & $\begin{array}{l}\text { 4-ethyl-2-methoxy-phenol(p- } \\
\text { ethylguaiacol) }\end{array}$ & $\mathrm{C} 9 \mathrm{H} 12 \mathrm{O} 2$ & 152 & 0.90 & 0.38 & 0.59 & 0.47 \\
\hline & 2,6-dimethoxy-phenol & $\mathrm{C} 8 \mathrm{H} 10 \mathrm{O} 3$ & 154 & 0.85 & 0 & 0.47 & 0.51 \\
\hline & 2-methoxy-4-propyl-phenol & $\mathrm{C} 10 \mathrm{H} 14 \mathrm{O} 2$ & 166 & 0.39 & 0 & 0 & 0 \\
\hline & $\begin{array}{l}\text { 4-methoxy-3-(methoxymethyl)- } \\
\text { phenol }\end{array}$ & $\mathrm{C} 9 \mathrm{H} 12 \mathrm{O} 3$ & 168 & 0.56 & 0.46 & 0 & 0.46 \\
\hline & Butylated hydroxytoluene & $\mathrm{C} 15 \mathrm{H} 24 \mathrm{O}$ & 220 & 2.11 & 6.71 & 11.06 & 10.86 \\
\hline \multirow{2}{*}{ Esters } & $\begin{array}{l}\text { 2-propenoic acid, 3-(1-acetyl-2,2- } \\
\text { dimethylcyclopentyl)-, methyl ester }\end{array}$ & $\mathrm{C} 13 \mathrm{H} 20 \mathrm{O} 3$ & 224 & 0.41 & 0.36 & 0.82 & 1.04 \\
\hline & $\begin{array}{l}\text { Terephthalic acid, } \\
\text { ethylhexyl)ester }\end{array}$ & $\mathrm{C} 24 \mathrm{H} 38 \mathrm{O} 4$ & 390 & 23.32 & 11.01 & 0 & 0 \\
\hline \multirow{4}{*}{ Others } & 2-chloro-2-methyl-butane & $\mathrm{C} 5 \mathrm{H} 11 \mathrm{Cl}$ & 106 & 8.43 & 22.50 & 17.70 & 13.11 \\
\hline & $\begin{array}{l}\text { 2,5,8-trimethyl-1,2,3,4-tetrahydro-1- } \\
\text { naphthol }\end{array}$ & $\mathrm{C} 13 \mathrm{H} 18 \mathrm{O}$ & 190 & 0.32 & 0 & 0 & 0 \\
\hline & 9-hexadecenoic acid & $\mathrm{C} 16 \mathrm{H} 30 \mathrm{O} 2$ & 254 & 0.65 & 0.42 & 0.63 & 0.65 \\
\hline & (Z)-9-octadecenamide & $\mathrm{C} 18 \mathrm{H} 35 \mathrm{NO}$ & 281 & 6.31 & 8.56 & 2.89 & 10.46 \\
\hline Total & & & & 80.84 & 77.04 & 67.23 & 70.08 \\
\hline
\end{tabular}


Table 3 Mass, Carbon and Hydrogen balances of HTL products resulting from conversion of the four feedstocks at three reaction temperatures: $0 \mathrm{~min}, 10 \mathrm{~min}$ and $20 \mathrm{~min}$

\begin{tabular}{|c|c|c|c|c|c|c|c|c|c|c|c|c|c|}
\hline & & \multicolumn{3}{|c|}{ Feed A } & \multicolumn{3}{|c|}{ Feed B } & \multicolumn{3}{|c|}{ Feed C } & \multicolumn{3}{|c|}{ Feed D } \\
\hline & & $0 \mathrm{~min}$ & $10 \mathrm{~min}$ & $20 \mathrm{~min}$ & $0 \mathrm{~min}$ & $10 \mathrm{~min}$ & $20 \mathrm{~min}$ & $0 \mathrm{~min}$ & $10 \mathrm{~min}$ & 20 min & $0 \mathrm{~min}$ & $10 \mathrm{~min}$ & $20 \mathrm{~min}$ \\
\hline \multirow{3}{*}{$\begin{array}{c}\text { Mass } \\
\text { Balance } \\
\text { (wt.\% db) }\end{array}$} & Solids & $14.2 \pm 1.1$ & $15.5 \pm 0.9$ & $19.0 \pm 1.7$ & $20.3 \pm 0.1$ & $21.6 \pm 2.3$ & $22.6 \pm 1.9$ & $26.0 \pm 4.0$ & $31.8 \pm 0.4$ & $32.7 \pm 1.8$ & $40.5 \pm 4.7$ & $37.1 \pm 4.9$ & $40.3 \pm 2.2$ \\
\hline & Biocrude & $49.8 \pm 2.1$ & $34.5 \pm 4.4$ & $38.4 \pm 2.3$ & $29.3 \pm 4.0$ & $28.5 \pm 2.6$ & $30.1 \pm 4.3$ & $39.0 \pm 5.3$ & $31.0 \pm 0.2$ & $29.8 \pm 0.6$ & $44.2 \pm 3.9$ & $43.0 \pm 1.9$ & $40.2 \pm 6.4$ \\
\hline & Gas* & $19.6 \pm 1.3$ & $21.4 \pm 3.4$ & $24.5 \pm 4.0$ & $15.2 \pm 2.9$ & $14.9 \pm 4.4$ & $16.7 \pm 0.3$ & $11.2 \pm 0.3$ & $15.2 \pm 0.8$ & $19.0 \pm 5.0$ & $14.6 \pm 0.7$ & $17.8 \pm 3.6$ & $16.1 \pm 1.6$ \\
\hline \multirow{3}{*}{$\begin{array}{c}\text { Carbon } \\
\text { Balance } \\
\text { (wt.\% db) }\end{array}$} & Solids & $19.1 \pm 1.3$ & $22.4 \pm 1.9$ & $26.9 \pm 0.9$ & $30.4 \pm 0.4$ & $32.8 \pm 4.6$ & $35.7 \pm 1.6$ & $18.4 \pm 4.0$ & $18.4 \pm 4.1$ & $17.8 \pm 2.0$ & $26.1 \pm 0.9$ & $22.2 \pm 1.3$ & $26.1 \pm 1.9$ \\
\hline & Biocrude & $69.0 \pm 2.4$ & $49.7 \pm 4.9$ & $55.1 \pm 5.6$ & $41.4 \pm 5.3$ & $39.3 \pm 5.4$ & $43.5 \pm 7.0$ & $56.6 \pm 1.8$ & $48.2 \pm 1.3$ & $46.5 \pm 0.9$ & $61.7 \pm 1.7$ & $58.6 \pm 1.2$ & $58.8 \pm 3.3$ \\
\hline & Gas* & $12.3 \pm 1.0$ & $14.8 \pm 1.4$ & $15.7 \pm 2.1$ & $9.6 \pm 1.4$ & $10.7 \pm 3.3$ & $13.0 \pm 0.8$ & $7.3 \pm 0.1$ & $10.9 \pm 0.4$ & $14.8 \pm 4.2$ & $9.7 \pm 0.4$ & $12.5 \pm 2.5$ & $11.7 \pm 0.9$ \\
\hline \multirow{3}{*}{$\begin{array}{c}\text { Hydrogen } \\
\text { Balance } \\
\text { (wt.\% db) }\end{array}$} & Solids & $8.1 \pm 0.6$ & $9.8 \pm 1.1$ & $11.9 \pm 1.5$ & $10.8 \pm 1.1$ & $11.9 \pm 1.6$ & $11.9 \pm 0.4$ & $9.9 \pm 1.6$ & $7.9 \pm 3.0$ & $7.8 \pm 0.4$ & $15.0 \pm 0.7$ & $12.9 \pm 1.4$ & $12.7 \pm 2.1$ \\
\hline & Biocrude & $68.0 \pm 2.9$ & $47.1 \pm 5.3$ & $55.1 \pm 2.2$ & $39.8 \pm 5.1$ & $39.4 \pm 3.0$ & $43.0 \pm 5.3$ & $57.5 \pm 2.8$ & $49.1 \pm 0.1$ & $47.5 \pm 1.8$ & $64.7 \pm 6.0$ & $65.3 \pm 2.4$ & $62.7 \pm 11.3$ \\
\hline & Gas* & $4.9 \pm 1.3$ & $10.3 \pm 1.7$ & $7.4 \pm 0.9$ & $4.1 \pm 1.0$ & $9.8 \pm 3.2$ & $13.6 \pm 1.7$ & $9.3 \pm 0.7$ & $14.0 \pm 1.7$ & $18.0 \pm 2.8$ & $11.8 \pm 0.3$ & $14.3 \pm 2.7$ & $16.8 \pm 2.4$ \\
\hline
\end{tabular}


Figure 1 Gas composition as function of the HTL reaction time

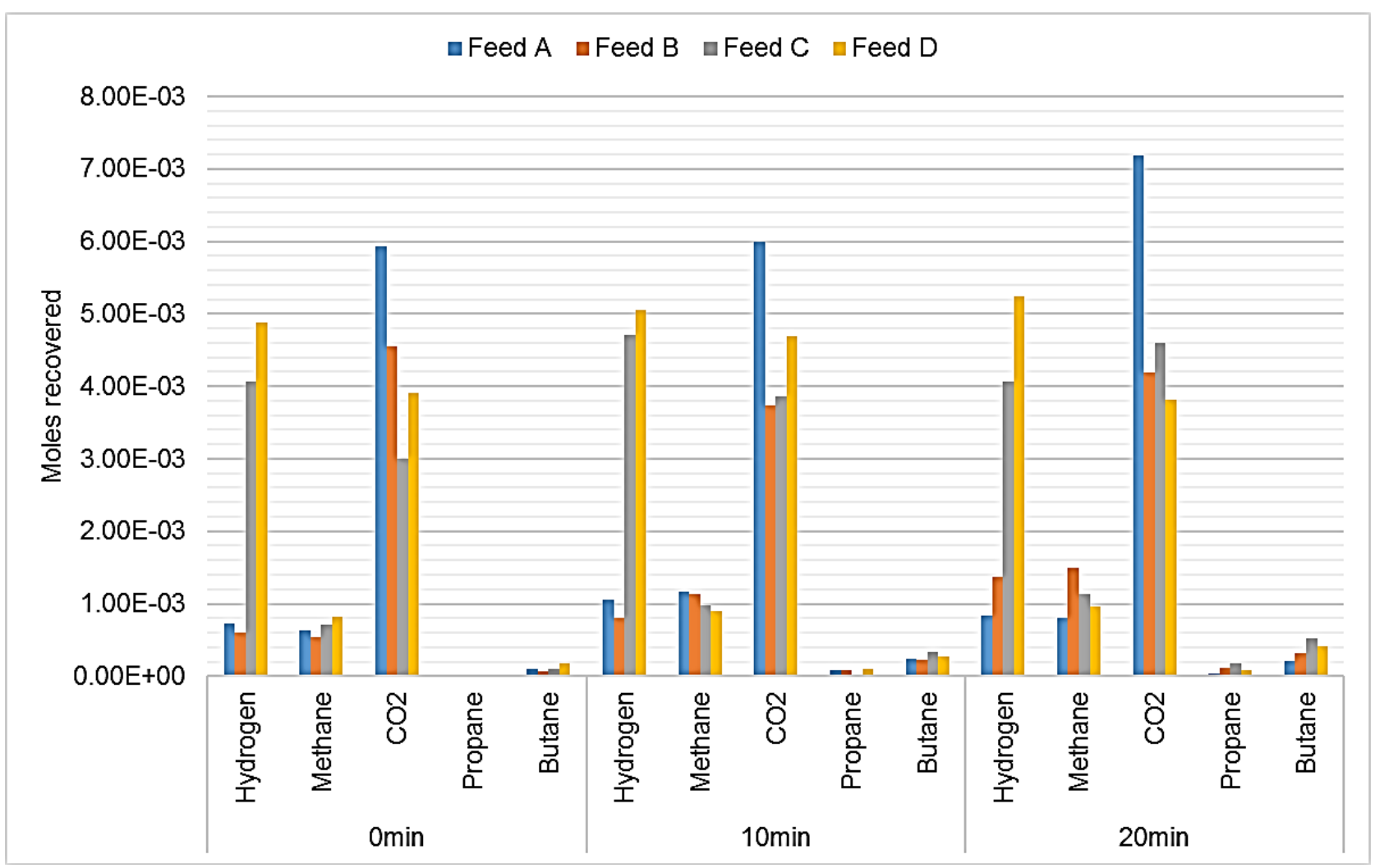

